\title{
TUNNELING CONDUCTIVITY OF MANGANITES WITH NEGATIVE MAGNETORESISTANCE
}

\author{
Yu.V. Medvedev ${ }^{a}$, V.Yu. Tarenkov ${ }^{a}$, V.M. Svistunov ${ }^{a}$, \\ A.V. AbalosheV ${ }^{b}$, I.S. Abalosheva ${ }^{b}$ and S.J. LeWandowsKi ${ }^{b}$ \\ ${ }^{a}$ Donetsk Physical-Technical Institute \\ of the National Academy of Sciences of Ukraine \\ R. Luxemburg 72, 340114 Donetsk, Ukraine \\ ${ }^{b}$ Instytut Fizyki PAN, Al. Lotników 32/46, 02-668 Warszawa, Poland
}

\begin{abstract}
Tunneling investigations of ferromagnetic $\mathrm{La}_{0.6} \mathrm{Sr}_{0.4} \mathrm{MnO}_{3}$ ceramic samples showing negative magnetoresistance effect were carried out in two types of junctions: symmetric "break junctions" and asymmetric ceramicsinsulator-metal junctions. The results are consistent with the presence of small ferromagnetic clusters in the insulating barriers of both types of junctions and suggest a two-phase state realized by impurity mechanism of phase separation.
\end{abstract}

PACS numbers: 75.70.Pa

During the last years electron tunneling in doped ferromagnetic manganites is used for the investigation of low-field magnetoresistance (MR) effect in polycrystalline structures [1] and in junctions on the base of epitaxial films [2]. Tunneling junctions composed of lanthanum manganite electrodes are proposed as spin valve devices [3].

In the present work we report on the investigation of spin-dependent electron tunneling in two types of junctions on the basis of $\mathrm{La}_{0.6} \mathrm{Sr}_{0.4} \mathrm{MnO}_{3}$ ceramics made by solid-state synthesis. The first type was a symmetric "break junction", and the second one an asymmetric ceramics-insulator-metal junction, formed by pressing a lead pellet into the surface of a ceramic sample.

The resistance of ceramic samples before the elaboration of the junctions was in the $0.8-2 \Omega$ range at room temperature. The resistance exhibited a maximum near $360 \mathrm{~K}$, associated with metal-insulator phase transition. The resistance of current and potential contacts was approximately $10^{-7} \Omega / \mathrm{cm}^{2}$.

In manganites, as well as in high- $T_{\mathrm{c}}$ superconductors, oxygen concentration plays fundamental role in the appearance of the metal-insulator transition. Mechanical deformations of the sample in the process of junction preparation can be the cause of oxygen escape from the surface of the perturbed region. The oxygen deficient region becomes then an insulator. In such a way a tunneling junction is formed between metal and ceramics or between ceramics and ceramics. The resistance of such tunneling contacts was found to be in the range of 100-300 $\Omega$. 
The existence of superconducting lead energy gap $\Delta=1.4 \mathrm{meV}$ (Fig. 1a) on the plots of tunneling conductance at $4.2 \mathrm{~K}$ confirms the fact that tunneling is the sole mechanism of charge transfer in the asymmetric $\mathrm{La}_{0.6} \mathrm{Sr}_{0.4} \mathrm{MnO}_{3}-\mathrm{Pb}$ junction. The experiments have shown that MR of this junction does not depend on the state of the lead electrode.

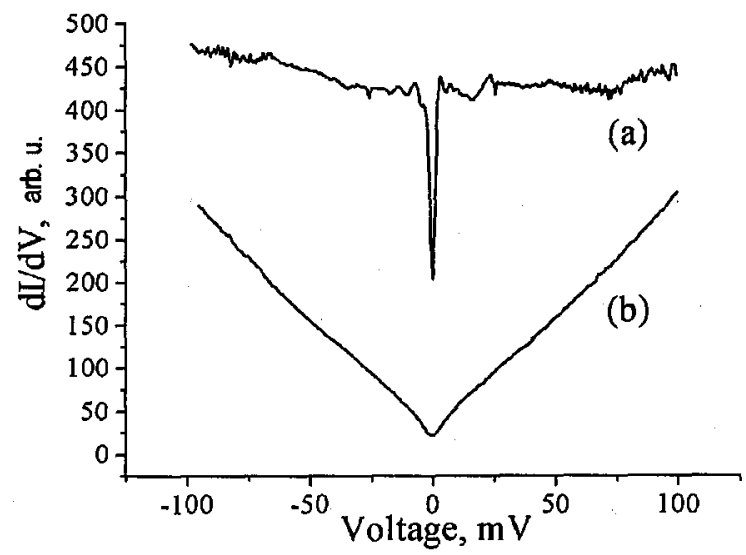

Fig. 1. Tunneling conductance at $4.2 \mathrm{~K}$ of two types of junctions: (a) asymmetric $\mathrm{La}_{0.6} \mathrm{Sr}_{0.4} \mathrm{MnO}_{3}-\mathrm{Pb}$, (b) symmetric $\mathrm{La}_{0.6} \mathrm{Sr}_{0.4} \mathrm{MnO}_{3}-\mathrm{La}_{0.6} \mathrm{Sr}_{0.4} \mathrm{MnO}_{3}$.

Conductance $\sigma$ of both types of junctions was characterized by linear dependence on bias voltage $V$ (Fig. 1b). This anomaly has been observed in tunneling experiments involving high- $T_{\mathrm{c}}$ superconductors [4].

The influence of magnetic field on the conductance of $\mathrm{La}_{0.6} \mathrm{Sr}_{0.4} \mathrm{MnO}_{3}-$ $\mathrm{La}_{0.6} \mathrm{Sr}_{0.4} \mathrm{MnO}_{3}$ symmetric junction (Fig. 2) was observed at all values of bias voltage. The plot of conductivity for junction in a nonzero external field was displaced upwards relative to the plot which has been obtained in zero field. The maximal relative sensitivity of the conductance to magnetic field was observed at $V=0$ and decreased with increasing voltage (cf. insert in Fig. 2).

The conductance $\sigma(H)$ of the investigated tunnel junctions at $77 \mathrm{~K}$ had a minimum in weak magnetic fields (Fig. 3). For tunneling junctions formed from ferromagnetic electrodes the presence of such a minimum is explained by the spin valve effect and the difference in the coercive fields of the electrodes. However, "break junction" is a symmetric tunneling junction in the sense of magnetic properties of the electrodes, while lead is not a magnetic material. Nevertheless, the behavior of $\sigma(H)$ of these two different structures is identical. This fact confirms that the MR mechanism in both cases has the same nature.

The data shown in Fig. 3 exhibit the characteristic MR behavior of tunneling junctions with small ferromagnetic clusters in the insulating layer, as described in Refs. $[5,6]$. We suppose, therefore, that we are dealing with a process analogous to spin-dependent electron tunneling across a barrier containing localized ferromagnetic centers. 


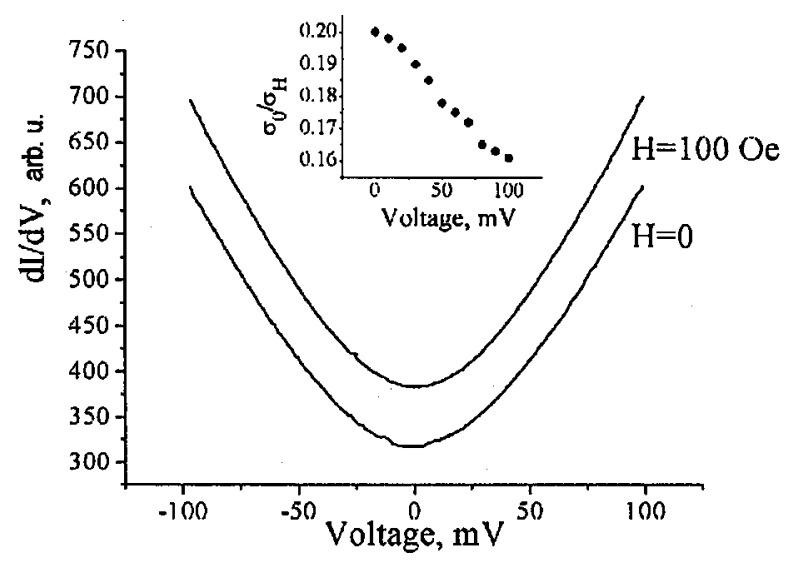

Fig. 2. Tunneling conductance of $\mathrm{La}_{0.6} \mathrm{Sr}_{0.4} \mathrm{MnO}_{3}-\mathrm{La}_{0.6} \mathrm{Sr}_{0.4} \mathrm{MnO}_{3}$ junction at two values of the applied magnetic field: $H=0 \mathrm{Oe}$ and $H=100 \mathrm{Oe}$. Insert shows the normalized junction conductance $\sigma_{H=0} / \sigma_{H=100}$.

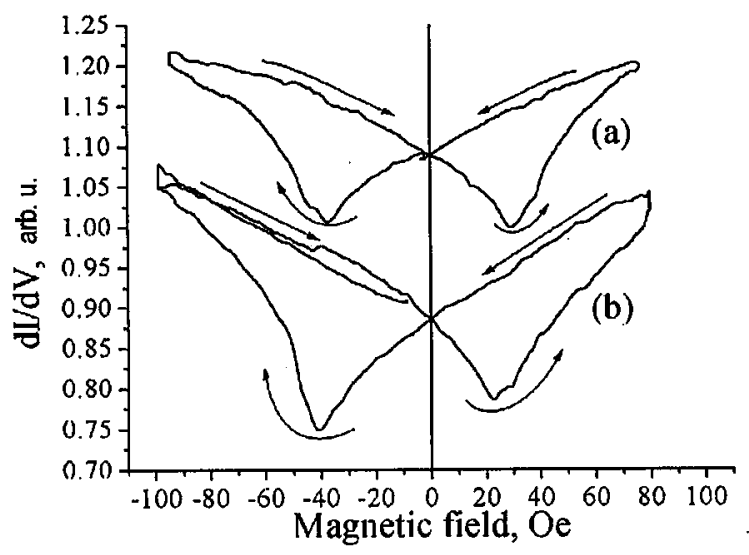

Fig. 3. Dependence of tunneling conductance on external magnetic field at $77 \mathrm{~K}$ : (a) $\mathrm{La}_{0.6} \mathrm{Sr}_{0.4} \mathrm{MnO}_{3}-\mathrm{Pb}$ junction, (b) $\mathrm{La}_{0.6} \mathrm{Sr}_{0.4} \mathrm{MnO}_{3}-\mathrm{La}_{0.6} \mathrm{Sr}_{0.4} \mathrm{MnO}_{3}$ junction. Arrows show direction of field change.

Oxygen deficit in $\mathrm{La}_{0.6} \mathrm{Sr}_{0.4} \mathrm{MnO}_{3}$ near the junction area is the most likely explanation of the appearance of ferromagnetic microregions in the barrier layer of both types of the investigated junctions. According to Ref. [7], if a verage oxidation of Mn ions of a given region is smaller than 3.2, magnetization $M$ of this region will be lowered in comparison to the other parts of the sample. Moreover, such perturbations of the stoichiometry lead to the appearance of a structure of ferromagnetic clusters in insulator matrix. The coercive field of such two-phase barrier layer differs from the coercive field of the electrodes.

The observed weak dependence of MR on the bias voltage (see insert in Fig. 2) is in agreement with the results of Ref. [5]. In the case of $\mathrm{CoFe}-\mathrm{Al}_{2} \mathrm{O}_{3}-\mathrm{Co}$ 
junctions [8] MR decreases almost 20 times in the range from 0 to $0.7 \mathrm{~V}$, whereas in the case of $\mathrm{Co}-\mathrm{Al}_{2} \mathrm{O}_{3}$-Co samples containing a layer of Co inclusions in the oxide of aluminum, MR decreases twice in the same range of voltage [5]. Finally, in the case of $(\mathrm{Au}-\mathrm{Cr})-\mathrm{Co}_{36} \mathrm{Al}_{22} \mathrm{O}_{42}-(\mathrm{Au}-\mathrm{Cr})$ junctions $\mathrm{MR}$ almost does not depend on $V$. This diversity of results is related to the complex structure of the junction, which results in the redistribution of voltage drop between granules and electrodes. Probably the strong dependence of MR on $V$ in classical junctions is related to the existence of a fine structure in spin-polarized density of states [5]. In junctions which contain ferromagnetic microregions in the insulating layer the fine structure can be smeared as a result of tunneling through resonant levels in the barrier.

The model of the two-phase state of La manganites constitutes one of the attempts to explain the nature of colossal MR near the temperature of phase transition in these compounds [9]. For stoichiometric $\mathrm{La}_{0.6} \mathrm{Sr}_{0.4} \mathrm{MnO}_{3}$ the two-phase state can arise from electronic separation of phases. Its existence is consistent with the fact [10] that spontaneous magnetization $M_{\mathrm{s}}$ at $4.2 \mathrm{~K}$ of $\mathrm{La}_{0.7} \mathrm{Sr}_{0.3} \mathrm{MnO}_{3}$ is less than the expected $M_{\mathrm{c}}$ corresponding to complete ferromagnetic ordering, $M_{\mathrm{s}} / M_{\mathrm{c}}=0.95$.

Results of Ref. [11] also confirm the possibility of realization of a two-phase state. According to the results of the present work the two-phase state in non-stoichiometric compounds is realized by impurity mechanism of phase separation.

This work was supported by the Committee for Scientific Research under grants 2 PO3B 09512 and 2 PO3B 14814.

\section{References}

[1] H.Y. Hwang, S.-W. Cheong, N.P. Ong, B. Batlogg, Phys. Rev. Lett. 77, 2041 (1996).

[2] Yu Lu, X.W. Li, G.Q. Gong, Gang Xiao, A. Gupta, P. Lecoeur, J.Z. Sun, Y.Y. Wang, V.P. Dravid, Phys. Rev. B 54, R8357 (1996).

[3] M. Viret, M. Drouet, J. Nassar, J.P. Contour, C. Fermon, A. Fert, Europhys. Lett. 39, 545 (1997).

[4] F. Sharifi, A. Pargellis, R.C. Dynes, Phys. Rev. Lett. 67, 509 (1991).

[5] L.P. Schelp, A. Fert, F. Fettar, P. Holody, S.F. Lee, J.L. Maurice, F. Petroff, A. Vaures, Phys. Rev. B 56, R5747 (1997).

[6] K. Inomata, H. Ogiwara, Y. Saito, K. Yusu, K. Ichihara, Jpn. J. Appl. Phys. 36, L1380 (1997).

[7] H.L. Ju, Hyunchul Sohn, J. Magn. Magn. Mater. 167, 200 (1997).

[8] J.S. Moodera, L.R. Kinder, T.M. Wong, R. Meservey, Phys. Rev. Lett. 74, 3273 (1995).

[9] E.L. Nagaev, Usp. Fiz. Nauk 8, 833 (1996).

[10] A. Urushibara, Y. Morimoto, T. Arima, A. Asamitsu, G. Kido, Y. Tokura, Phys. Rev. B 51, 14103 (1995).

[11] I. Koroleva, P.B. Demin, A.M. Balbashov, JETP Lett. 6, 474 (1997). 